\title{
Dual-polarized antenna design integrated with metasurface and partially reflective surface for $5 \mathrm{G}$ communication
}

\author{
Zhilong Li, Yajie Mu, Jiaqi Han ${ }^{*}$, Xiaohe Gao, and Long Li
}

Key Laboratory of High Speed Circuit Design and EMC of Ministry of Education, School of Electronic Engineering, Xidian University, Xi'an 710071, PR China

Received: 22 April 2020 / Accepted: 5 August 2020

\begin{abstract}
A design of electrical down-tilt dual-polarized base station antenna array (BSAA) for 5G communication applications is presented in this paper, which is realized by integrating with reconfigurable reflective metasurface and partially reflective surface (PRS). By controlling the varactor diodes which are inserted into the reflective elements, we can adjust the mainlobe direction of BSAA. Moreover, the PRS over the array is utilized to construct Fabry-Perot (FP) cavity with reflective metasurface and ground plane. Based on this design approach, a $1 \times 6$ dual-polarized BSAA operating from $3.4 \mathrm{GHz}$ to $3.6 \mathrm{GHz}$ is designed and fabricated. Simulated and measured results show that the gain is enhanced about $2.56 \mathrm{~dB}$ by PRS while side lobe level (SLL) is less than $-20 \mathrm{~dB}$. The mainlobe of the antenna array can be adjusted accurately within $\pm 5^{\circ}$ for beam down-tilt. The cross polarization discrimination (XPD) is less than $-40 \mathrm{~dB}$.
\end{abstract}

Keywords: Metasurface / partially reflective surface / electrical down-tilt / base station antenna / Fabry-Perot cavity

\section{Introduction}

Base station antenna has been greatly developed since the use of printed circuit board technology [1]. And a lot of novel designs of base station antenna are proposed during these years. Most of these designs have outstanding advantages, such as the ultra-wide bandwidth, high front to back ratio (FBR), high isolation, low profile and so on [2-4]. Dual-polarized T shaped printed dipole is widely used in base station applications due to the advantages of low-cost and ease of fabrication [5]. Double layered printed T-shaped dipole is proposed for decreasing the XPD [6]. Multiple frequency resonance is adjusted closer together to achieve broadband in printed planar dipoles. Furthermore, parasitic microstrip line and arc-shaped baffle plates are adopted to improve the radiation pattern and isolation [7-13].

Base station is getting more and more intelligent and reconfigurable [14]. In order to increase system capacity and spectrum usage efficiency, Massive MIMO technology and frequency reuse technology are employed in base station research [15]. However, co-channel interference caused by frequency reuse between adjacent cellular systems should be prevented without blind area in many situations [16]. One of the most feasible methods to solve

\footnotetext{
* e-mail: jqhan@xidian.edu.cn
}

the problem and to increase signal coverage is the electrical down-tilt of the BSAA. Phase shifters are usually integrated with the feeding network to compensate the required phases of the antenna cells, thus realize the downtilt of the antenna array [17]. But this approach accompanied by high cost, low accuracy and signal distortion. So, a new method for base station to achieve electrical down-tilt is necessary.

Metasurface as the planar metamaterial is widely used in antennas owing to the excellent performance in manipulating electromagnetic waves. Especially, high impedance surface (HIS), electromagnetic bandgap (EBG) structure and frequency selective surface (FSS) are always adopted to construct in-phase reflective surface for low profile and high isolation in base station appliances [18,19]. Conventional metasurface can achieve special function, such as reflective coding metasurface combined with FP antenna to realize high-gain and low-RCS simultaneously [20]. Apart from that, active components such like PIN diodes, microelectromechanical systems (MEMS) and varactor diodes are integrated with the elements to form reconfigurable meta-surface. Novel designed reconfigurable PRS enables the patch antenna to realize low RCS and high gain in broadband [21].

In this paper, we present a new method to achieve the electrical down-tilt of the dual-polarized BSAA by means of reconfigurable reflective metasurface and PRS. 


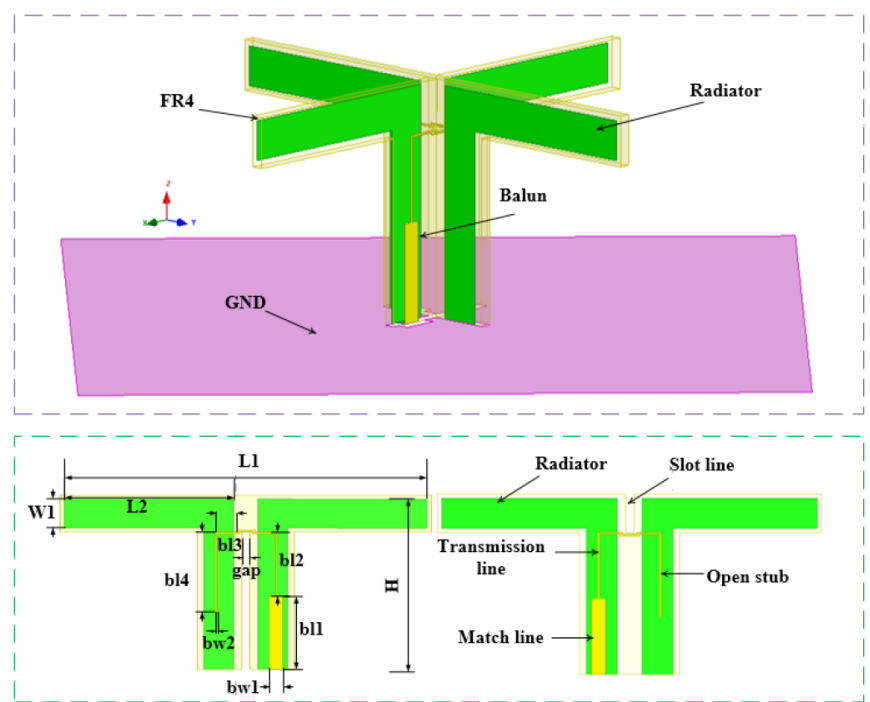

Fig. 1. Geometry of dual-polarized dipole and single-polarized dipole.

The outline of this paper is as follows. First, the design of the antenna element and reconfigurable reflective metasurface element is given in Section 2. Next, the design of the FP cavity is shown in Section 3. The concept of the BSAA down-tilt via reflective metasurface will be described in Section 4. Simulated and measured results of the whole structure will be presented in Section 5. Finally, conclusion is drawn in Section 6.

\section{Antenna element and reflective meta-surface}

\subsection{Antenna element}

The antenna element is a dual-polarized T-shaped printed dipole consisting of a radiator, a feed balun, slot line and ground plane. As shown in Figure 1, the feeding balun and radiator are printed on the front side and back side of an F4B substrate, the substrate is $1 \mathrm{~mm}$ thick while the dielectric constant and loss tangent are 2.65 and 0.001 respectively. The balun consists of a matching line, a transmission line and an open stub, they are critical to match the impedance of the dipole to $50 \Omega$, both of the baluns are bent to avoid intersect. The geometric parameters of the antenna are $\mathrm{L} 1=46 \mathrm{~mm}$, gap $=1 \mathrm{~mm}$, bl $1=9 \mathrm{~mm}, \quad$ bl $2=7.2 \mathrm{~mm}, \quad$ bl $3=3 \mathrm{~mm}, \quad$ bl $4=10.2 \mathrm{~mm}$, bw $1=1.7 \mathrm{~mm}, \mathrm{bw} 2=0.2 \mathrm{~mm}, \mathrm{~W} 1=3.6 \mathrm{~mm}, \mathrm{~L} 2=20.8 \mathrm{~mm}$ and $\mathrm{H}=21.6 \mathrm{~mm}$. The printed dipole is mounted (at center) above a ground plane with dimensions of $65 \mathrm{~mm} \times 65 \mathrm{~mm}$ and fed by a power splitter underneath the ground plane. Energy is coupled from balun to the radiator by slot line. Figure 2 shows the simulation results of the dual-polarized dipole antenna. As can be seen from Figure $2 \mathrm{a}$ that the center frequency of the designed element is $3.5 \mathrm{GHz}$, and the bandwidth of $\mathrm{S} 11<-15 \mathrm{~dB}$ is from 3.35 to $3.65 \mathrm{GHz}$. The $\mathrm{S} 21$ is about $-25 \mathrm{~dB}$ during the whole working frequency band. The radiation patterns in zoy-plane and zox-plane at $3.5 \mathrm{GHz}$

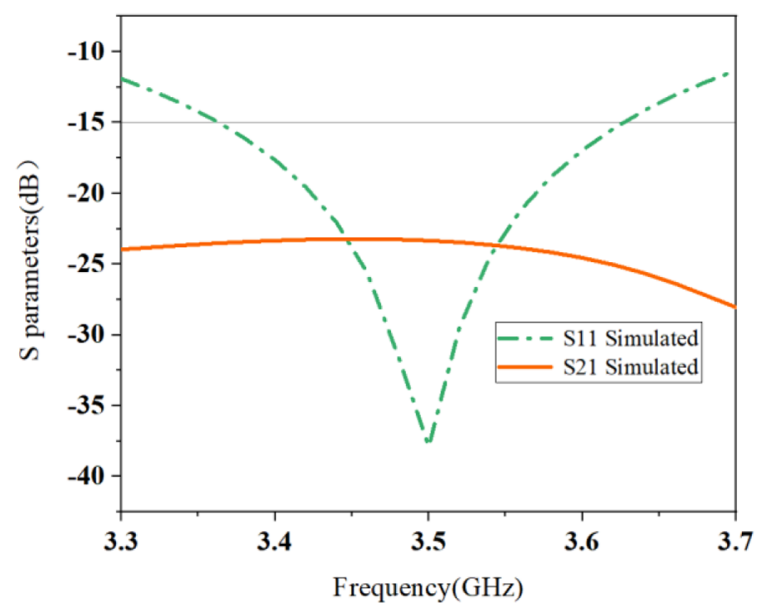

(a)
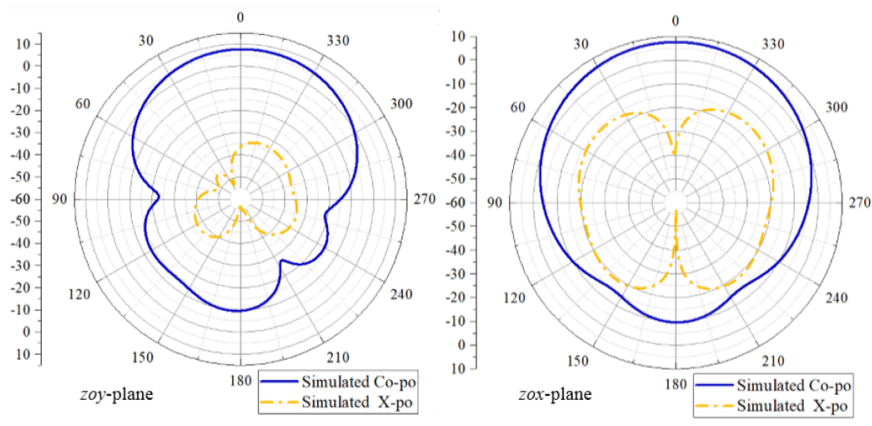

(b)

Fig. 2. (a) Simulated $\mathrm{S} 11$ and $\mathrm{S} 21$ of the dual polarized dipole element, (b) simulated radiation pattern at $3.5 \mathrm{GHz}$ in $z o y$-plane and $z o x$-plane.

for dual-polarized antenna are shown in Figure 2b. The half power bandwidth (HPBW) is $68.76^{\circ}$ in zoy-plane and $84^{\circ}$ in zox-plane, respectively, and the gain is $7.6 \mathrm{~dB}$ at $3.5 \mathrm{GHz}$. The XPD is more than $35 \mathrm{~dB}$ at $z$-axis, and $17 \mathrm{~dB}$ at $\pm 60^{\circ}$ off-from $z$-axis.

\subsection{Reflective element}

In general, reconfigurable reflective metasurface element is constructed by metallic patch and active components. Square loop is selected as metallic pattern of the reflective element. Geometric parameters and structure are depicted in Figure 3. The square loop and ground plane are printed on the top side and bottom side of F4B substrate. Metallic branch protrudes from the edge of the square loop and is connected to the ground plane through metallized hole. Varactor diodes are inserted into the gap of metallic branch, different reflection phase responses of metasurface elements are realized by controlling the states of the varactor diodes. The period of the cell is $d=55 \mathrm{~mm}$. Outside length of the square loop is $\mathrm{Lo}=21.5 \mathrm{~mm}$ while inside length is $\mathrm{Li}=10.75 \mathrm{~mm}$. The thickness of the $\mathrm{F} 4 \mathrm{~B}$ substrate is $\mathrm{t}=2 \mathrm{~mm}$, while relative permittivity and loss tangent are 2.65 and 0.001 respectively. 


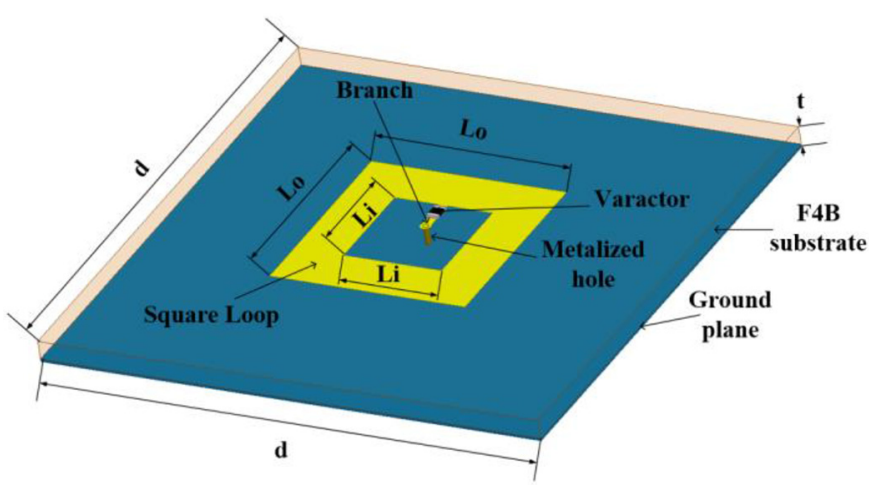

Fig. 3. Perspective view of reflective element.

\section{FP resonant cavity}

The electromagnetic waves coming from the antenna experience multiple reflections and transmissions within the FP resonance cavity formed by reconfigurable reflective metasurface and PRS. On behalf of the pure substrate has same reflection effect to different linear polarization waves, so the PRS made of pure substrate is adopted to reflect the forward beam of the BSAA back to the metasurface for further phase manipulation. As is shown in Figure 4a, the antenna element is mounted (at center) above two substrates. Two reflective elements are etched at both side of the antenna cell, and they are all printed on the top side of the upper substrate, the top side of the lower substrate is ground plane. The PRS is made of Rogers 6010 which the relative permittivity is 10.2 and loss tangent is 0.0023. It combined with the ground plane to form a FP resonance cavity. The distance of the PRS above the ground plane is determined by the reflective phase of the PRS, the specific value is $H=47.16 \mathrm{~mm}$, and $L=130 \mathrm{~mm}$ in this design. We found that the down-tilt angles based on the compensated phases which are obtained by adding periodic boundary to the reflective element are not accurate. It might be caused by the complicated phase distribution of the electromagnetic waves in the FP cavity. A new method to get the correct phase compensation is proposed in this paper. Antenna element with the PRS and two reflective cells are treated as a sub FP cavity. The states corresponding to the required phases are selected by the reflective element in the sub FP cavity to realize the down-tilt of the BSAA. Simulated reflective phase of far field electromagnetic waves is shown in Figure 4b. Varactor diodes show different capacitance while adding different bias voltages. The phase range of the far field electromagnetic waves radiated from the sub FP cavity is $85^{\circ}$ while turning the capacitance of the varactor from $0.63 \mathrm{pF}$ to 2.7 $\mathrm{pF}$, and thus the required phases of various down-tilt angles are obtained by adding different bias voltages to the varactor diodes in the metasurface from $0 \mathrm{~V}$ to $30 \mathrm{~V}$.

\section{Design and down-tilt of the BSAA}

Unlike phase shifters, they compensate the required phase of the antenna elements in microwave circuits, metasurface

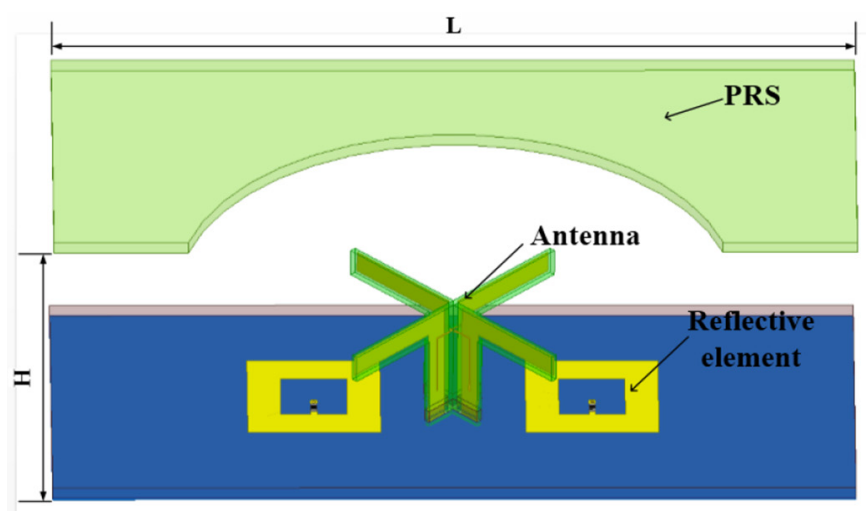

(a)

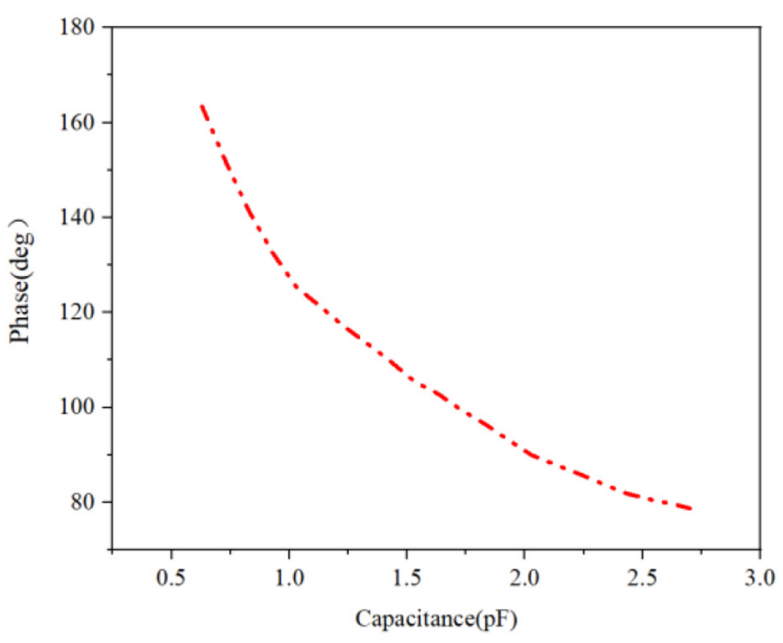

(b)

Fig. 4. (a) Perspective view of FP resonance cavity, (b) simulated result of phase response.

achieves the same effect in spatial field. The electrical down-tilt theory of the BSAA is derived from

$$
\begin{aligned}
\phi_{n} & =k_{0} d_{n} \\
& =k_{0}(n-1) d\left(\sin \theta_{0} \cos \varphi_{0}+\sin \theta_{0} \sin \varphi_{0}\right),
\end{aligned}
$$

where $\phi_{n}$ is the compensating phase of the reflective cells in the sub cavity, $k_{0}$ is wavenumber in free space, the distance between the adjacent antenna cells is $d=55 \mathrm{~mm}, \theta$ is the azimuth angle, and $\varphi$ is the elevation angle which is a constant in this design, $n$ is the number of the reflective elements.

In order to reduce the circuit loss of transmission line, a novel designed wideband 1 to 6 power splitter is proposed in this work to directly feed the BSAA without coaxial cables. As shown in Figure 5a, a three-section impedance transformer is adopted to achieve wideband performance of the power splitter. The specific values of three impedance matching lines are $43.3 \Omega, 74.8 \Omega$ and $70.7 \Omega$ respectively. Figure $5 \mathrm{~b}$ is the prototype of the power splitter, and measured results which are not given here for the brevity of the figures show that the circuit loss of the power splitter is less than $0.5 \mathrm{~dB}$. 


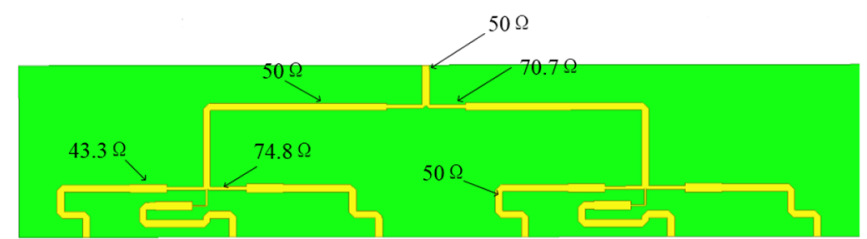

(a)

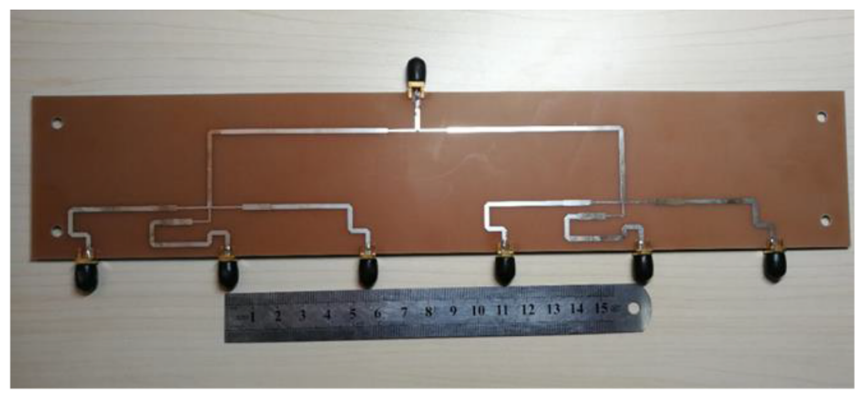

(b)

Fig. 5. (a) Geometry of the power splitter, (b) prototype of the power splitter.

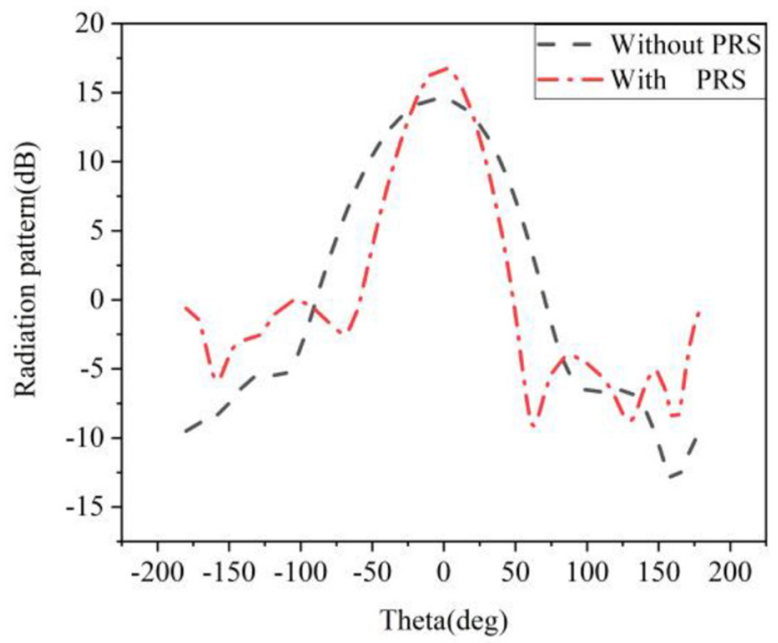

(a)

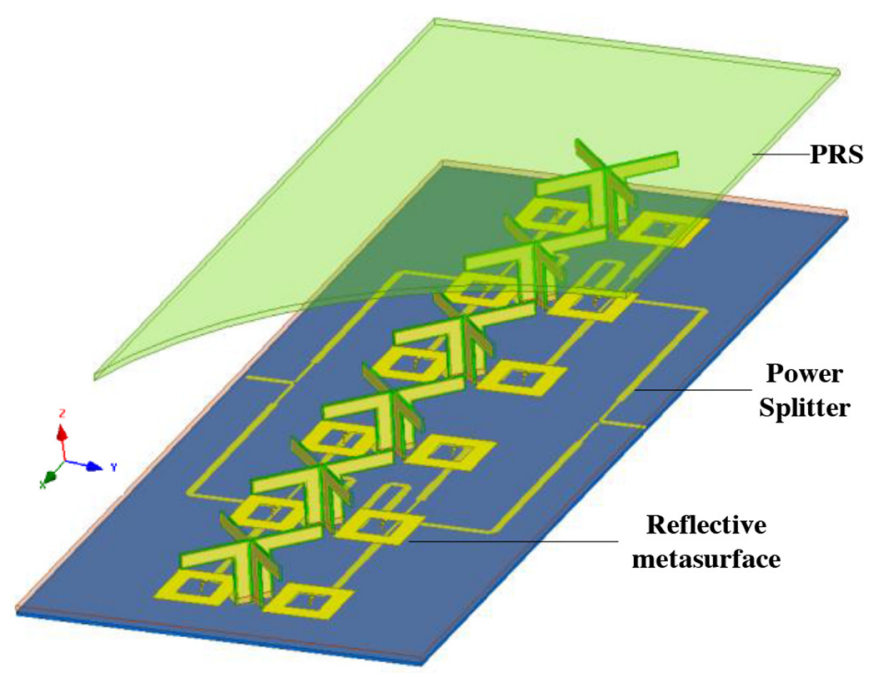

Fig. 6. Perspective view of the BSAA with PRS and feeding network.

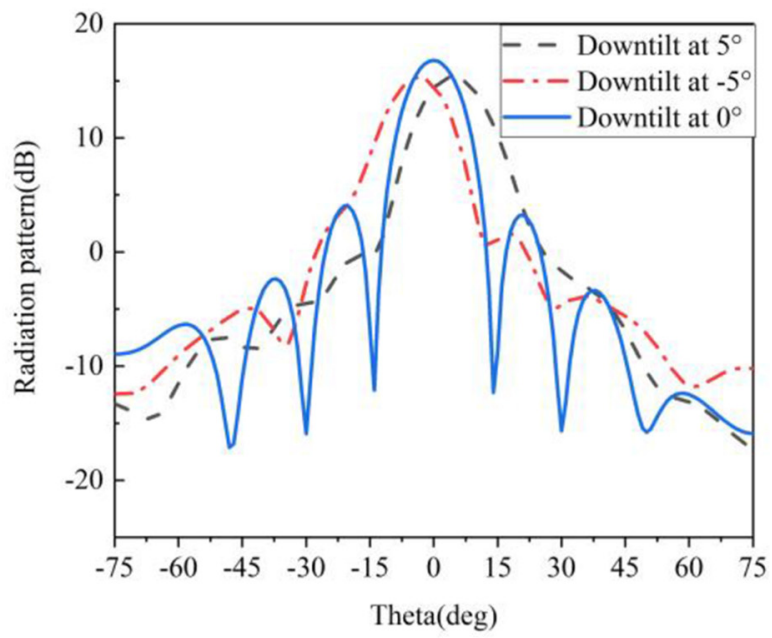

(b)

Fig. 7. (a) Simulated gain of the BSAA with or without PRS, (b) simulated down-tilted mainlobe of the BSAA at $0^{\circ}$ and $\pm 5^{\circ}$.

To demonstrate the potential applications of T-shaped dipole with the reconfigurable reflective elements and the PRS, we put these parts together to construct a novel designed BSAA which is excited by the power splitter. As shown in Figure 6, six base station antenna elements are arranged along $x$-axis to form a linear array with 12 reflective cells on the both sides. The dual-polarized BSAA which operates at $3.5 \mathrm{GHz}$ with $11.1 \%$ relative bandwidth is used as exciter. Feeding network consists of two novel designed wideband 1 to 6 power splitters which cling to the bottom layer of lower substrate is utilized to inspire the dual-polarized BSAA. Required phases of the meta-surface calculated from the formula (1) are adopted by the metasurface to realize the beam down-tilt of the BSAA.

In order to investigate the effects of the PRS on the performance of the proposed BSAA, we compared the simulation results of the BSAA with/without PRS. As can be seen from Figure 7a, the gain of the BSAA with PRS is $16.58 \mathrm{~dB}$, which is $2.56 \mathrm{~dB}$ higher than that without PRS, and the SLL is less than $-20 \mathrm{~dB}$. So we can conclude that the FP cavity formed by the PRS and reflective metasurface can not only reflect the forward beam back to the metasurface, but also can enhance the gain of the BSAA. The phase manipulation formula of down-tilt is concluded in Section 4. Required phase responses in Figure $4 \mathrm{~b}$ are adopted by the reflective elements in the FP cavity to achieve beam down-tilt of the BSAA with $1^{\circ}$ step. The simulated downtilt radiation pattern is shown in Figure 7b, for the brevity of the figure, we only choose to draw a few representative downtilt angles of the radiation pattern to demonstrate the performance of the BSAA. The mainlobe of the antenna array can be adjusted accurately within $\pm 5^{\circ}$ for beam down-tilt. The gain of the main-lobe is about 

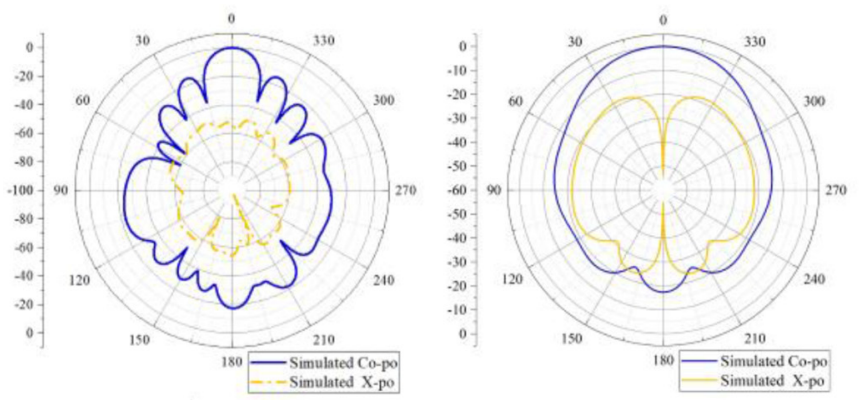

(a)
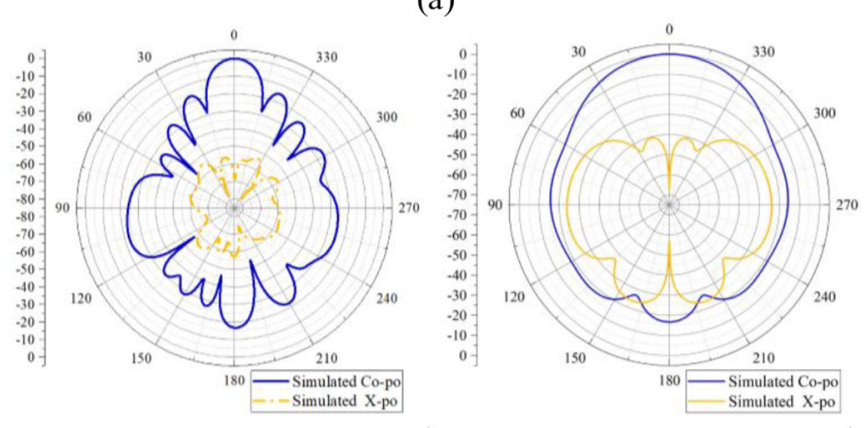

(b)
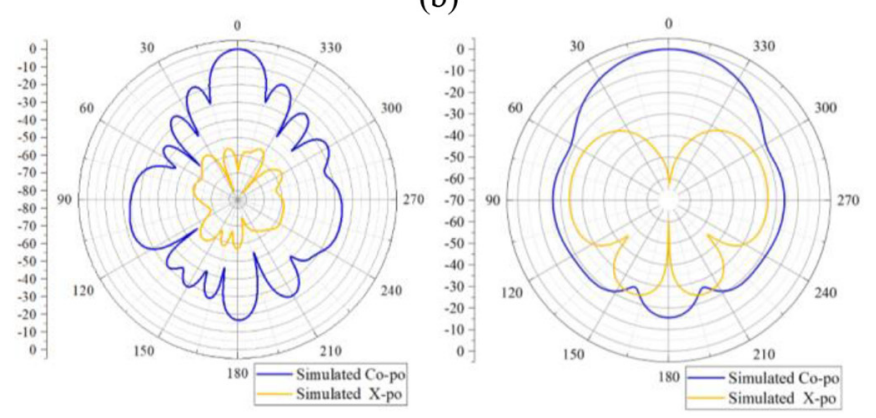

(c)

Fig. 8. Simulated co- and cross-polarized radiation patterns in zoy-plane and zox-plane, (a) $3.4 \mathrm{GHz}$, (b) $3.5 \mathrm{GHz}$, (c) $3.6 \mathrm{GHz}$.

$1.3 \mathrm{~dB}$ lower while down-tilt to $5^{\circ} /-5^{\circ}$. First, drop of the gain might be caused by the decreasing of the array factor while the main-lobe is at $5^{\circ} /-5^{\circ}$ according to antenna theory. On the other hand, both the ability of FP cavity to converge the oblique incident waves and the efficiency of the ground plane to reflect the electromagnetic waves were declined. The simulated co- and cross-polarized radiation patterns from $3.4 \mathrm{GHz}$ to $3.6 \mathrm{GHz}$ in zoy-plane and zoxplane are shown in Figure 8. It is obvious that the T-shaped dual-polarization antenna array has a stable gain across the entire working frequency band. We note that the gain of the whole structure in the working frequency band are $15.94 \mathrm{~dB}$ at $3.4 \mathrm{GHz}, 16.58 \mathrm{~dB}$ at $3.5 \mathrm{GHz}, 16.83 \mathrm{~dB}$ at $3.6 \mathrm{GHz}$. The HPBW are $43.3^{\circ}, 45.9^{\circ}, 50.53^{\circ}$ in $z o y$-plane, and $13.13^{\circ}, 13.4^{\circ}, 13.55^{\circ}$ in $z o x$-plane respectively, the normalized cross polarization of the BSAA are less than $-40 \mathrm{~dB}$ among the working frequency band. Figure 9 shows the simulated gain of the BSAA, and the gains for both polarizations are about $16.2 \pm 0.9 \mathrm{~dB}$ in the whole frequency band.

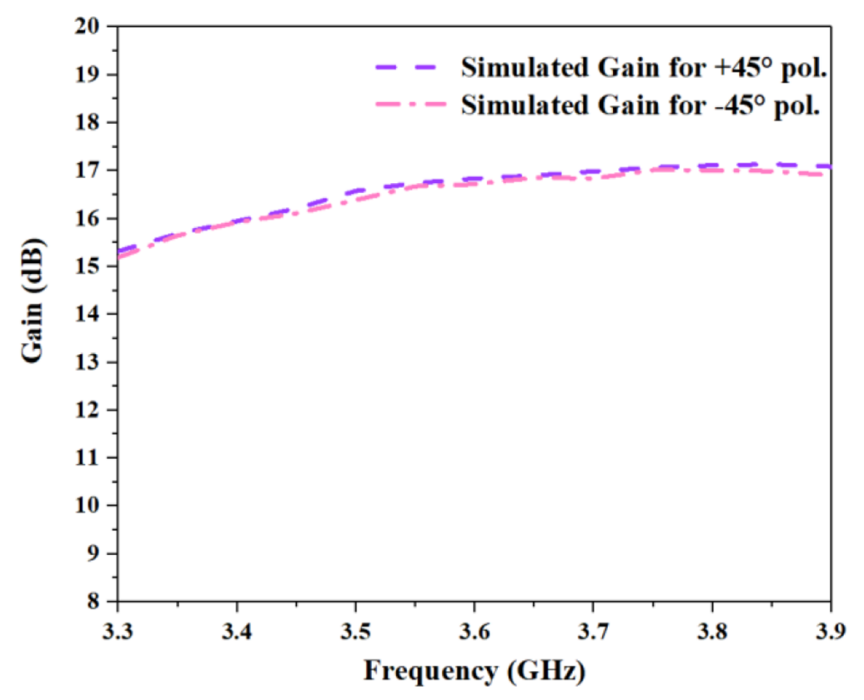

Fig. 9. Simulated gain of the proposed antenna array.

\section{Experimental results and analysis}

The novel designed BSAA occupies a size of $330 \times 55 \times$ $50 \mathrm{~mm}^{3}$. Figure 10 exhibits the photograph of the fabricated BSAA, the compared BSAA without PRS and reflective metasurface are depicted in Figure 10a. In order to evaluate the performance of the BSAA, S11 is measured by the vector network analyzer KEYSIGHT N9928A. As shown in Figure 10b, the radiation pattern of the BSAA is measured in the anechoic chamber. Considering the cost, the material of the PRS is replaced by FR4 substrate (relative permittivity and loss tangent are 4.4 and 0.005 respectively) with the thickness of $2 \mathrm{~mm}$. The measured S11 of the BSAA with / without PRS and reflective metasurface is depicted in Figure 10c. The measured S11 of the proposed antenna array is less than $-10 \mathrm{~dB}$ from $3.4 \mathrm{GHz}$ to $3.8 \mathrm{GHz}$, and less than $-40 \mathrm{~dB}$ at $3.48 \mathrm{GHz}$, it is slightly moving to the upper band compared to the simulated result. The frequency offset may be caused by changing the material of PRS.

Moreover, the influence of metallic control device should be considered to the loss of the measured result while offering bias voltages to the varactor diodes. In order to discuss the influence of the PRS and reconfigurable metasurface, compared antenna array is measured. The S11 of the compared antenna array is less than $-40 \mathrm{~dB}$ in $3.95 \mathrm{GHz}$, but it is worse than proposed antenna array from $3.4 \mathrm{GHz}$ to $3.6 \mathrm{GHz}$. Apparently, the PRS and reconfigurable metasurface are good for impedance matching of the BSAA. Figure 10d shows the normalized down-tilt radiation patterns of the BSAA at $0^{\circ},-5^{\circ}$ and $+5^{\circ}$, it can accurate tilt in $1^{\circ}$ step, and we only draw these three downtilt angles for the brevity of the figure. Due to symmetry of the structure, down-tilt angles from $0^{\circ}$ to $5^{\circ}$ can be realized by reverse the bias voltages of $-5^{\circ}$ to $0^{\circ}$ to reflective metasurface. Comparisons between the proposed antenna array and other antennas are listed in Table 1, and we can conclude that this paper creatively proposed a new method to realize the electrical down-tilt of the BSAA in 


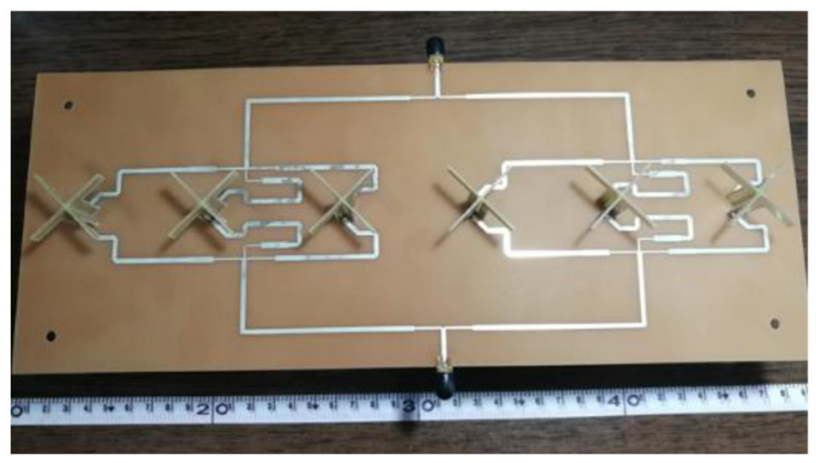

(a)

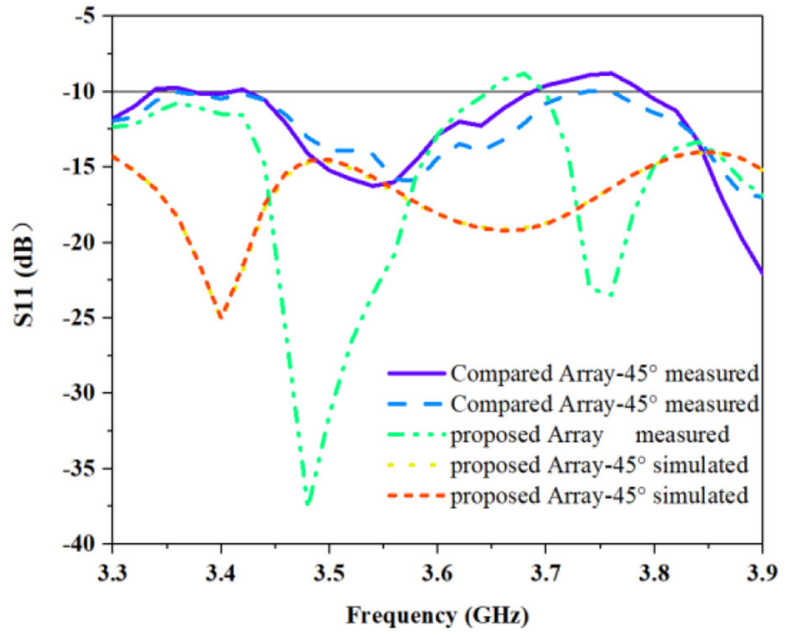

(c)

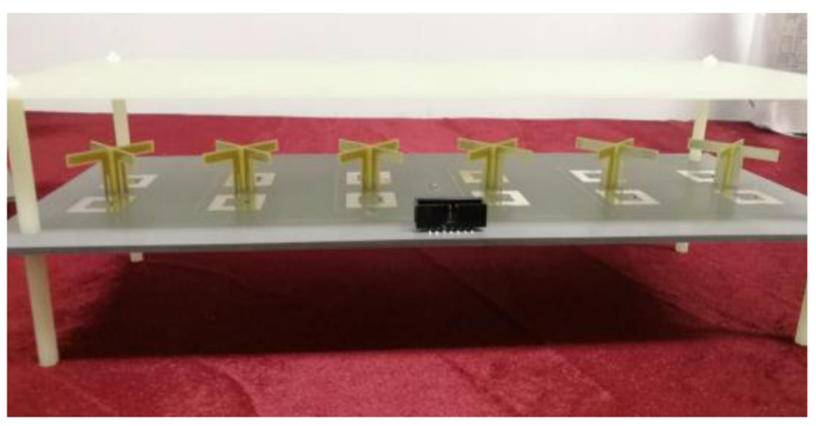

(b)

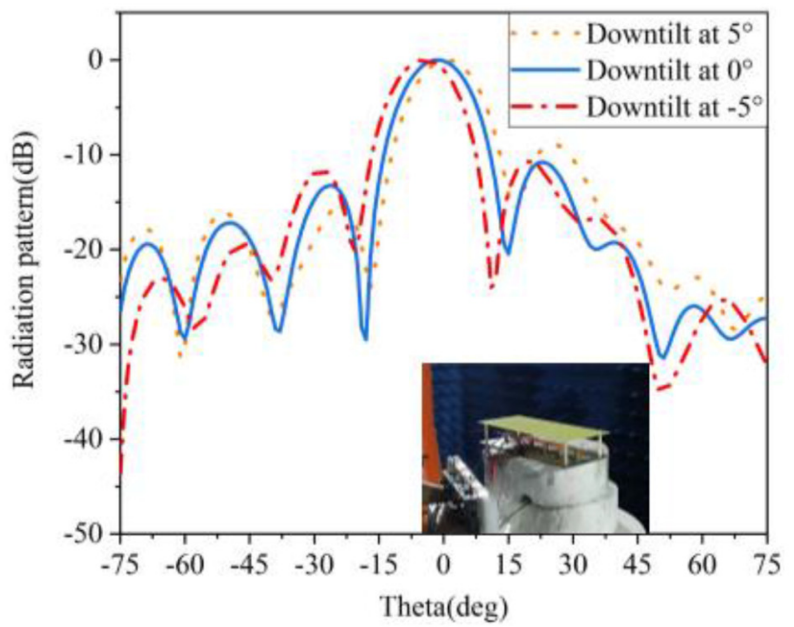

(d)

Fig. 10. (a) Fabricated compared BSAA, (b) fabricated BSAA with PRS, (c) measured and simulated S11 of the BSAA, (d) measured normalized down-tilt pattern of the BSAA at $0^{\circ}$ and $\pm 5^{\circ}$.

Table 1. Comparison of proposed antenna array for base station.

\begin{tabular}{lllll}
\hline References & $\mathrm{BW} / \mathrm{GHz}$ & Down-tilt angle & Polarization & Down-tilt method \\
\hline$[14]$ & $0.79-0.96 \& 1.71-2.17$ & $0-14^{\circ}$ & Dual-polarization & Phase shifter \\
& & $0-10^{\circ}$ & & \\
{$[17]$} & $0.83-0.9$ & $3-15^{\circ}$ & Single-polarization & Phase shifter \\
This work & $3.4-3.6$ & -5 to $5^{\circ}$ & Dual-polarization & Meta-surface \\
\hline
\end{tabular}

two directions by reconfigurable metasurface. All these results show good performance of the BSAA in electrical down-tilt, and the whole structure has enormous potential in $5 \mathrm{G}$ communication systems.

\section{Conclusion}

In this paper, we present a new method to realize the electrical down-tilted mainlobe of the dualpolarized BSAA operating at $3.5 \mathrm{GHz}$. First, a dualpolarized T-shaped printed dipole is introduced, and novel designed reconfigurable reflective cell is proposed to form a $2 \times 6$ metasurface. Then, required phases that can tilt the mainlobe of the BSAA are obtained by controlling the states of the reconfigurable reflective metasurface in the FP cavity. Finally, both simulated and measured result show that the mainlobe of the BSAA down-tilt accurately within $\pm 5^{\circ}$. The whole structure shows good mechanical characteristics such as easy to fabricate, compact structure, low cost, good stability and high accuracy. The number of the active components is greatly reduced in the metasurface. Compared with the traditional electrical down-tilt method, the active inter-modulation distortion to the RF signal radiating from the BSAA can be greatly reduced by tailoring the electromagnetic waves in spatial field instead of in microwave circuit. It shows enormous potential for $5 \mathrm{G}$ services in modern mobile communication system. 


\section{References}

1. E. Levine, S. Shtrikman, D. Treves, Double-sided printed arrays with large bandwidth, IEE Proc. H Microw. Antennas Propag. 135, 54-59 (1988)

2. Q. He, B. Wang, J. He, Wideband and dual-band design of a printed dipole antenna, IEEE Antennas Wirel. Propag. Lett. 7, 1-4 (2008)

3. B.Q. Wu, K. Luk, A broadband dual-polarized magnetoelectric dipole antenna with simple feeds, IEEE Antennas Wirel. Propag. Lett. 8, 60-63 (2009)

4. J.-R. Bayard, M.E. Cooley, D.H. Schaubert, Analysis of infinite arrays of printed dipoles on dielectric sheets perpendicular to a ground plane, IEEE Trans. Antennas Propagat. 39, 1722-1732 (1991)

5. J. Perruisseau-Carrier, T.W. Hee, P.S. Hall, Dual-polarized broadband dipole, IEEE Antennas Wireless Propag. Lett. 2, 310-312 (2003)

6. Z. Zhou, S. Yang, Z. Nie, A novel broadband printed dipole antenna with low cross-polarization, IEEE Trans. Antennas Propag. 55, 3091-3093 (2007)

7. Y. Cui, R. Li, P. Wang, Novel dual-broadband planar antenna and its array for $2 \mathrm{G} / 3 \mathrm{G} / \mathrm{LTE}$ base stations, IEEE Trans. Antennas Propag. 61, 1132-1139 (2013)

8. Y. Gou, S. Yang, J. Li, Z. Nie, A compact dual-polarized printed dipole antenna with high isolation for wideband base station applications, IEEE Trans. Antennas Propag. 62, 4392-4395 (2014)

9. Y. Cui, R. Li, H. Fu, A broadband dual-polarized planar antenna for $2 \mathrm{G} / 3 \mathrm{G} / \mathrm{LTE}$ base stations, IEEE Trans. Antennas Propag. 62, 4836-4840 (2014)

10. Q. Chu, D. Wen, Y. Luo, A broadband $\pm 45^{\circ}$ dual-polarized antenna with Y-shaped feeding lines, IEEE Trans. Antennas Propag. 63, 483-490 (2015)

11. H. Huang, Y. Liu, S. Gong, A dual-broadband, dualpolarized base station antenna for $2 \mathrm{G} / 3 \mathrm{G} / 4 \mathrm{G}$ applications, IEEE Antennas Wireless Propag. Lett. 16, 1111-1114 (2017)
12. A. Alieldin et al., A triple-band dual-polarized indoor base station antenna for $2 \mathrm{G}, 3 \mathrm{G}, 4 \mathrm{G}$ and sub-6 $\mathrm{GHz} 5 \mathrm{G}$ applications, IEEE Access 6, 49209-49216 (2018)

13. M. Li, X. Chen, A. Zhang, A.A. Kishk, Dual-polarized broadband base station antenna backed with dielectric cavity for 5G communications, IEEE Antennas Wireless Propag. Lett. 18, 2051-2055 (2019)

14. Y. He, W. Tian, L. Zhang, A novel dual-broadband dualpolarized electrical downtilt base station antenna for $2 \mathrm{G} / 3 \mathrm{G}$ applications, IEEE Access 5, 15241-15249 (2017)

15. R.M.C. Mestrom, T.J. Coenen, A.B. Smolders, Adaptive downtilt for cellular base stations, in 2012 International ITG Workshop on Smart Antennas (WSA), Dresden, 2012, pp. 162-167

16. G. Wilson, Electrical downtilt through beam-steering versus mechanical downtilt (base station antennas), in [1992 Proceedings/ Vehicular Technology Society 42nd VTS Conference - Frontiers of Technology, Denver, CO, USA, 1992, Vol. 1, pp. 1-4

17. H. Khodabakhshi, J. Rashed-Mohassel, F. Arazm, Design of an electrical downtilt base station antenna, in 2005 IEEE International Symposium on Microwave, Antenna, Propagation and EMC Technologies for Wireless Communications, Beijing, 2005, Vol. 1, pp. 148-152

18. Z. Nie, H. Zhai, L. Liu, J. Li, D. Hu, J. Shi, A dual-polarized frequency-reconfigurable low-profile antenna with harmonic suppression for 5G application, IEEE Antennas Wireless Propag. Lett. 18, 1228-1232 (2019)

19. Y. Zhu, Y. Chen, S. Yang, Decoupling and low-profile design of dual-band dual-polarized base station antennas using frequency-selective surface, IEEE Trans. Antennas Propag. 67, 5272-5281 (2019)

20. L. Zhang, H.T. Wu, T.J. Cui, A low-RCS and high-gain partially reflecting surface antenna based on coding metasurface, in 2017 Sixth Asia-Pacific Conference on Antennas and Propagation (APCAP), Xi'an, 2017, pp. 1-3

21. W. Pan, C. Huang, P. Chen, X. Ma, C. Hu, X. Luo, A lowRCS and high-gain partially reflecting surface antenna, IEEE Trans. Antennas Propag. 62, 945-949 (2014)

Cite this article as: Zhilong Li, Yajie Mu, Jiaqi Han, Xiaohe Gao, Long Li, Dual-polarized antenna design integrated with metasurface and partially reflective surface for 5G communication, EPJ Appl. Metamat. 7, 3 (2020) 